\title{
The normal response to prolonged passive head up tilt testing
}

\author{
M E V Petersen, T R Williams, C Gordon, R Chamberlain-Webber, R Sutton
}

\begin{abstract}
Objective-To define the responses to head up tilt in a large group of normal adult subjects using the most widely employed protocol for tilt testing.

Methods-127 normal subjects aged 19-88 years (mean (SD), 49 (20) years) without a previous history of syncope underwent tilt testing at $60^{\circ}$ for 45 minutes or until syncope intervened. Blood pressure monitoring was performed with digital photoplethysmography, providing continuous, non-invasive, beat to beat heart rate and pressure measurements.

Results-13\% of subjects developed vasovagal syncope after a mean (SD) tilt time of 31.7 (12.4) minutes (range 8.5-44.9 minutes). Severe cardioinhibition during syncope was observed less often than is reported in patients investigated for syncope. There were no differences in the age or sex distributions of subjects with positive or negative outcomes, or in the proportions with cardioinhibitory and vasodepressor vasovagal syncope compared with previously reported patient populations. Subjects with negative outcomes showed age related differences in heart rate and blood pressure behaviour throughout tilt.

Conclusions-False positive results with tilting appear to be common. This has important implications for the use of diagnostic tilt testing. The magnitude of the heart rate and blood pressure changes observed during negative tilts largely invalidates previously suggested criteria for abnormal non-syncopal outcomes.

(Heart 2000;84:509-514)
\end{abstract}

Keywords: syncope; head up tilt; postural hypotension

Prolonged head up tilt is now widely employed as a diagnostic test in patients with syncope. ${ }^{1}$ The most commonly observed positive outcome is vasovagal (neurocardiogenic) syncope. ${ }^{2}{ }^{3}$ Postural hypotension, excessive or inadequate heart rate responses, and psychogenic syncope have also been considered abnormal responses. ${ }^{3-7}$ Vasovagal outcomes during tilt have also been observed in healthy volunteers without a history of syncope. ${ }^{89}$ Therefore, before attributing diagnostic significance to tilt induced vasovagal responses in patients with clinical episodes of syncope, an acceptable specificity must first be defined. ${ }^{10}{ }^{11}$ This is influenced by the method of tilt employed and thus needs to be defined for each of the many protocols used in clinical practice. ${ }^{1} 1012$

Intravascular instrumentation, isoprenaline administration, longer duration and greater angles of tilt, and the use of saddle or parachute supports increase the rate of vasovagal responses in normal subjects and may compromise the specificity of such an outcome in patient populations. ${ }^{1} 911$ 13-16 Passive tilt protocols in which there is no intravascular instrumentation and no drug administration, and which are performed with footboard supports, are widely perceived to have much greater specificity. ${ }^{1}{ }^{10} 14$ This conclusion is supported by the seven studies employing passive tilt protocols of at least 30 minutes' duration which have included control subjects. ${ }^{314}{ }^{17-21}$ Three of these studies included controls with heart disease, hypertension, or subjects undergoing investigation for gastroenterological symptoms, and collectively $6 \%$ of these subjects showed vasovagal syncope. ${ }^{14} 1819$ In the remaining four studies, including 56 in normal controls, only one positive outcome was observed. ${ }^{3} 172021$

Despite these data there is cause for uncertainty. ${ }^{18}$ Relatively few normal subjects have been tested, and the $1.8 \%$ incidence of vasovagal syncope is considerably lower than might have been expected given the $10 \%$ incidence observed in two early studies, which included twice as many healthy subjects and employed only 20 minutes of tilt. ${ }^{822}$ Furthermore, the study of Natale and colleagues reported an $8-20 \%$ rate of syncope or presyncope with hypotension in healthy volunteers during 20 minutes of passive tilt at angles of $60^{\circ}$ to $80^{\circ} .{ }^{15}$ These are considerable disparities and suggest the need for further clarification of the specificity of the passive tilt protocols that are employed clinically.

No study of normal subjects has fully characterised the nature of the positive or negative responses observed. It is thus uncertain whether the vasovagal responses differ from those observed in patients. Furthermore, in the absence of such data, the suggested criteria for abnormal but non-syncopal outcomes observed during tilt remain speculative..$^{3-7}$

Our purpose in this study was to clarify these issues by defining the incidence and the nature of positive responses, and by describing the heart rate and blood pressure behaviour during negative responses, in a large group of well characterised normal adult controls undergoing the most widely recommended passive tilt test protocol. ${ }^{1423}$ 
Table 1 The VASIS (vasovagal international study) classification of vasovagal syncope

\begin{tabular}{ll}
\hline VASIS class & Definition of VASIS class \\
\hline Type 1 (mixed) & $\begin{array}{l}\text { Syncope is characterised by a fall in both blood pressure and } \\
\text { heart rate. The fall in blood pressure precedes the fall in heart } \\
\text { rate. The heart rate falls by more than } 10 \% \text { but does not fall to } \\
\text { less than } 40 \text { beats/min for more than } 10 \text { seconds } \\
\text { Syncope is characterised by a fall in both blood pressure and } \\
\text { heart rate. The fall in blood pressure precedes the fall in heart } \\
\text { rate. The minimum heart rate is less than } 40 \text { beats/min for } \\
\text { more than } 10 \text { seconds, or asystole occurs for more than } 3 \\
\text { Type 2a (cardioinhibitory) }\end{array}$ \\
$\begin{array}{l}\text { seconds } \\
\text { Tyncope is characterised by a fall in both blood pressure and } \\
\text { heart rate. The blood pressure falls to hypotensive levels }(<80 \\
\text { mm Hg) only after or coincident with a rapid and severe fall in } \\
\text { heart rate. The minimum heart rate is less than } 40 \text { beats/min } \\
\text { for more than } 10 \text { seconds, or asystole occurs for more than }\end{array}$ \\
$\begin{array}{l}3 \text { seconds } \\
\text { Syncope is characterised by a fall in blood pressure. The heart } \\
\text { rate does not fall by more than } 10 \% \text { from the maximum rate } \\
\text { observed during tilt }\end{array}$ \\
\hline
\end{tabular}

\section{Methods}

SUBJECT SELECTION

Subjects were recruited through advertisement and received no payment for their participation. Potential subjects were interviewed before the tilt test. They were eligible for inclusion if they were aged 18 years or older, and had no past history of fainting or unconsciousness, cardiovascular or neurological disease, diabetes, or any serious current illness. Subjects were excluded if they were taking any regular drugs other than the oral contraceptive pill, or showed any abnormality on examination or on a 12 lead ECG. None of the subjects had previously undergone tilt testing. All gave informed consent for the tilt test, and the study had local ethics committee approval.

TILT TEST PROTOCOL

Tilt testing was performed between 0900 and 1500 hours in a dedicated laboratory with subdued lighting and ambient temperature of $20-22^{\circ} \mathrm{C}$. Subjects were required to have fasted for four hours before the tilt. The test protocol involved a $60^{\circ}$ head up tilt with a footboard support for up to 45 minutes (negative outcome), or until syncope or extreme and intolerable symptoms intervened (positive outcome). ${ }^{14}$ The motorised tilt table achieved $60^{\circ}$ of tilt over 20 seconds. During the test, subjects were restrained by two Velcro straps placed around the legs and waist, and conversation, other than reporting symptoms, was discouraged.

Continuous and non-invasive beat to beat heart rate and blood pressure measurements were recorded. Data were obtained for a supine resting period of 12 minutes (resting), throughout the period of tilt, and for two minutes following return to the supine position (post-tilt). Heart rate data were obtained as a series of RR intervals with a resolution of $1 \mathrm{~ms}$. The blood pressure measurements were achieved by digital photoplethysmography (Finapres, Ohmeda, Englewood, Colorado, USA), a method we have previously described during tilt testing. ${ }^{24}{ }^{25}$ The times at which tilt up and tilt down occurred, at which symptoms were reported, and when syncope or presyncope interrupted the test were entered into the data record on-line during the test.
DATA PROCESSING

The data from each subject were reviewed and edited manually to remove artefacts. The heart rate and blood pressure data were then averaged for the resting period, for each minute of tilt, and in the post-tilt period.

Subjects were divided by age into three groups: younger than 35 years, 35-69 years, and 70 years or older. The blood pressure and heart rate of the subjects with negative outcomes in each group were compared.

Positive vasovagal outcomes were defined as cardioinhibitory if the minimum heart rate during syncope fell to $<60$ beats $/ \mathrm{min}$, and vasodepressor if the minimum heart rate was 60 beats/min or more. ${ }^{3}$ They were also classified according to the VASIS (vasovagal international study) criteria, which involve an assessment of the temporal relation between blood pressure fall and heart rate fall, as well as consideration of the absolute degree of bradycardia (table 1$).^{5}$

\section{STATISTICAL ANALYSIS}

Grouped data are expressed as mean (SD). When data did not conform to a normal distribution the median is provided. Statistical comparisons between grouped data were done using paired and non-paired $t$ tests. Categorical data were analysed using a $\chi^{2}$ test with Yates correction.

\section{Results}

One hundred and twenty seven normal subjects (mean (SD) age, 49 (20) years, range 19-88 years) underwent tilt testing, of whom $47(37 \%)$ were male and $80(63 \%)$ female; 111 subjects $(87 \%)$ completed 45 minutes of tilt without syncope or presyncope. None of these subjects reported symptoms. There were 16 positive outcomes (13\%). There were no differences in the age $(\mathrm{p}=0.76)$ or sex $(p=0.82)$ distributions of the positive and negative outcomes.

\section{POSITIVE OUTCOMES}

All positive outcomes resulted from profound hypotension accompanied to some degree by a fall in heart rate, and were thus considered vasovagal in origin. According to conventional classification, 11 of the positive outcomes were cardioinhibitory $(69 \%)$ and five were vasodepressor $(31 \%)$. With respect to the VASIS classification, 14 of the positive outcomes were type $1(88 \%)$, one type $2 \mathrm{a}(6 \%)$, and one type $2 \mathrm{~b}(6 \%)$.

Syncope occurred at a mean of 31.7 (12.4) minutes (range 8.5-44.9 minutes). Only three positive outcomes occurred during the first 20 minutes of tilt. Symptoms were reported before syncope in 15 of the subjects (94\%), with a median time from symptoms to syncope of 0.8 minutes (range 0.1-5.1 minutes).

NEGATIVE OUTCOMES

Significant age related differences were observed in the blood pressure and heart rate responses during negative tilts.

Within the first minute of tilt there were highly significant increases in systolic and 


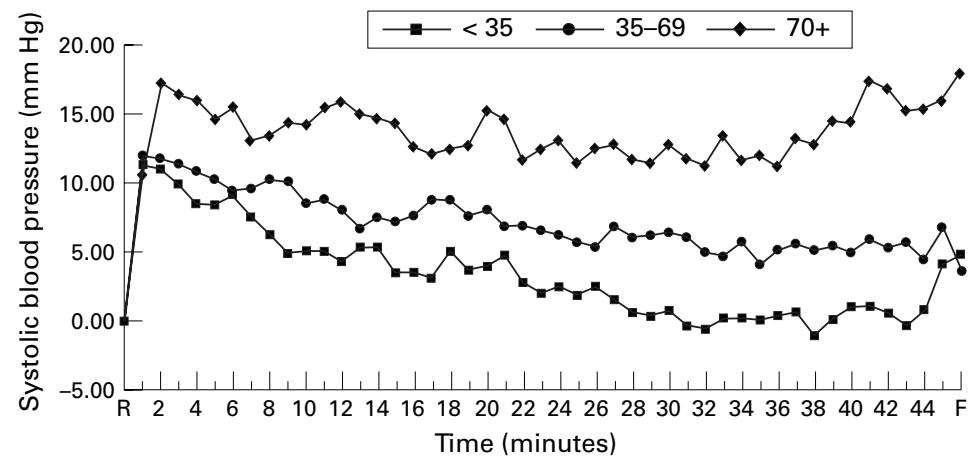

Figure 1 Change in systolic pressure $(\mathrm{mm} \mathrm{Hg})$ during tilt with respect to resting supine pre-tilt systolic pressure.

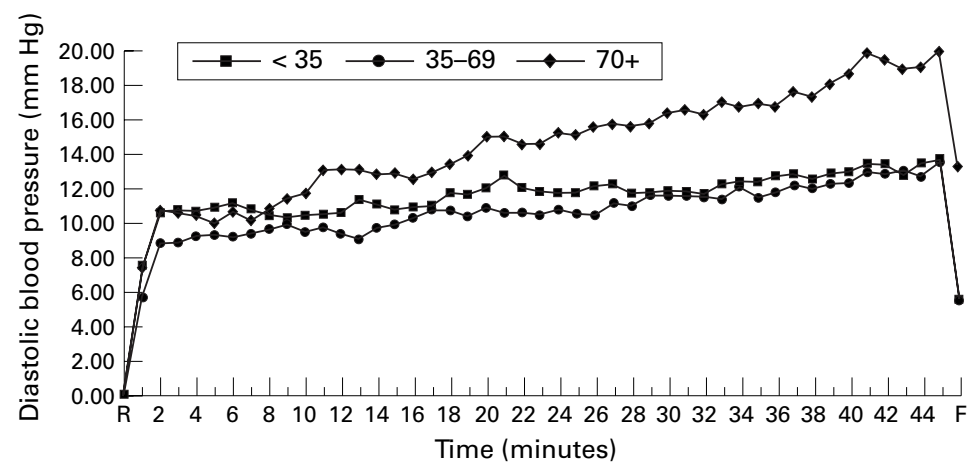

Figure 2 Change in diastolic pressure ( $\mathrm{mm} \mathrm{Hg}$ ) during tilt with respect to resting supine pre-tilt diastolic pressure.

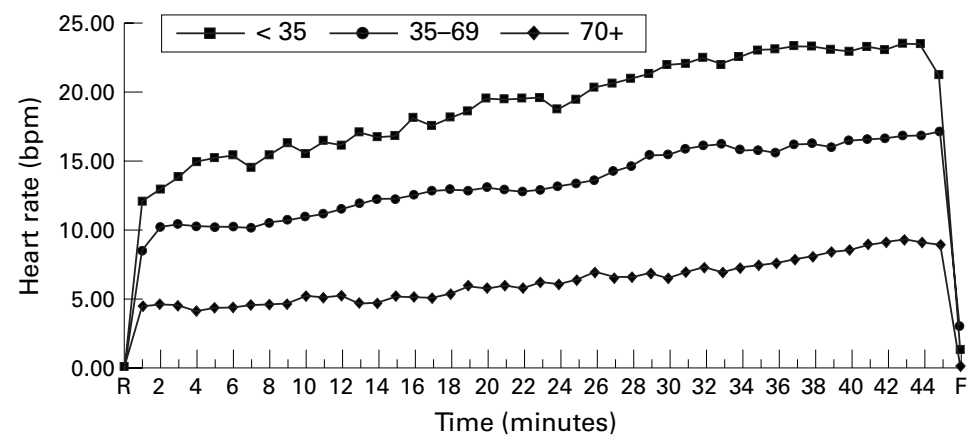

Figure 3 Change in heart rate (beats/min) during tilt with respect to resting supine pre-tilt heart rate.

diastolic pressure in each age group $(p<0.001)$. Thereafter systolic pressure drifted downwards towards resting levels while diastolic pressure slowly increased. In the $70+$ age group there was a further increase in systolic pressure from about 30 minutes, while in the younger age groups the downward trend continued (figs 1 and 2). Systolic pressures were significantly lower in the younger age groups than in the $70+$ group at rest $(\mathrm{p}<0.01$, $<35$ v 70+; $\mathrm{p}<0.05,35-69 \vee 70+$ ) and throughout tilt $(\mathrm{p}<0.01,<35$ and 35-69v $70+$ ). No differences between the age groups were observed in the diastolic pressures until the later stages of tilt, when diastolic pressures were significantly lower in the $<35$ group than in the $70+$ group $(\mathrm{p}<0.05)$.

A significant increase in heart rate was observed in the first minute of tilt $(\mathrm{p}<0.001$ for each age group). Subsequently, the heart rate increased gradually throughout the test (fig 3). The magnitude of the initial heart rate increase was greater in the $<35$ age group than in the older groups $(\mathrm{p}<0.001)$, and in the 35-69 age group than in the $70+$ group $(\mathrm{p}<0.001)$. The secondary heart rate increase was greater in the $<35$ age group than in the 35-69 group $(p<0.01)$, and in both younger age groups than in the $70+$ group $(\mathrm{p}<0.001)$.

\section{Discussion}

This study shows that during the most widely recommended passive tilt protocol, normal controls with no previous history of syncope or fainting had a $13 \%$ rate of vasovagal outcome, occurring at all ages. All positive outcomes were vasovagal and no subject with a negative outcome reported symptoms. Significant age related differences in the heart rate and blood pressure responses during negative outcomes were observed. These responses are relevant if abnormal but non-syncopal outcomes during tilt are to be defined.

\section{PREVIOUS TILT STUDIES INVOLVING CONTROL}

\section{SUBJECTS}

Vasovagal syncope during head up tilt testing of healthy young volunteers was shown over 50 years ago. ${ }^{8}$ To our knowledge there have been 23 published studies in which control subjects have been subjected to head up tilt of at least $60^{\circ}$ with a duration of at least 15 minutes and without the simultaneous infusion of drugs. \begin{tabular}{l}
38 \\
\hline
\end{tabular} 11 $^{13-22}$ 26-33 Without regard to any of the methodological differences in these studies, $11.2 \%$ of controls (101 of 900) developed vasovagal syncope. Thus it was established long before the use of tilt testing became fashionable in patients with unexplained syncope that, given a sufficient orthostatic stress, normal controls faint, and false positive outcomes were likely to be a problem in the interpretation of positive responses in patients.

These studies also show important differences in the incidence of vasovagal responses when different protocols are used. In the eight studies involving intravascular instrumentation, $22 \%$ of subjects (40 of 180 ) experienced vasovagal syncope $(\mathrm{p}<0.0001 v$ those without instrumentation), an observation in keeping with that of Stevens. ${ }^{13}$ In the 15 studies without instrumentation, the rate of vasovagal outcomes in those performed with saddle or parachute supports was significantly greater than in those with footboard supports $(12 \%$ v 5\%, $\mathrm{p}=0.001)$. The magnitude of this difference is probably underestimated in view of the considerably longer duration of tilt in the studies employing a footboard support.

In the seven studies where tilt was continued for at least 30 minutes and performed with a footboard support and without instrumentation, the incidence of vasovagal outcomes was $4.3 \%$ (six of 138). ${ }^{314}{ }^{17-21}$ The criteria used to select controls varied between studies and thus the majority of these subjects had cardiovascular disease or were under investigation for gastroenterological complaints. If these subjects are excluded, 56 normal controls from four studies remain, of whom only one $(1.8 \%)$ experienced vasovagal syncope during tilt. ${ }^{3172021}$ 
POSITIVE OUTCOME DATA IN THIS STUDY

The tilt protocol used in this study involves no intravascular instrumentation and employs a footboard support. It evolved as a result of earlier methodological studies. ${ }^{14}$ The limited experience of normal controls subjected to this protocol in earlier studies suggested an extremely low rate of vasovagal outcomes in this group. However, the $13 \%$ positive outcome rate observed in our study implies that, in the context of clinical practice, the protocol has a lower specificity than was previously assumed. ${ }^{1} 1023$

In studies of patients with unexplained syncope employing this or a closely similar tilt protocol, vasovagal syncope has been observed in $24-75 \% .^{3141720}$ Our data suggest that $13 \%$ of subjects might be expected to develop vasovagal syncope irrespective of their clinical history. Thus with respect to an attributable diagnosis, for patient populations with observed rates of vasovagal outcomes varying from $24 \%$ to $75 \%$ during tilt, some $17-54 \%$ might be considered to be false positives.

Vasovagal outcomes in our normal population occurred at all ages, as had been suggested by Lewis in his original description. ${ }^{34}$ Eleven were by definition cardioinhibitory and five vasodepressor-similar proportions to those observed in patient populations. ${ }^{314}$ By contrast, significant differences in the nature of the positive outcomes as classified by the VASIS system were observed in comparison with an earlier series of 182 consecutive patients with unexplained syncope or presyncope, where the same tilting protocol was used. ${ }^{55}$ Only two VASIS type 2 outcomes occurred in normal controls $(1.6 \%)$, compared with $24(12.1 \%)$ in the patient series $(p=0.002)$. No difference was observed in the frequency of the more prevalent VASIS type 1 outcome $(p=0.2)$. In the normal population no VASIS type 3 vasovagal syncope and no symptomatic nonvasovagal responses were observed, in contrast to patient populations, suggesting that these outcomes may be considered abnormal. ${ }^{3-7}$

These differences lend support to the concept of a more detailed classification of vasovagal outcomes during tilt and suggest that the more severe cardioinhibitory outcomes, which comprise the VASIS type 2 group, may be more specific to patients with recurrent clinical episodes of syncope.

NEGATIVE TILT TEST OUTCOMES

It has been suggested that in addition to syncope various other abnormal patterns of heart rate and blood pressure behaviour may be recognised during diagnostic tilt testing of patients with unexplained syncope..$^{3-63637}$ These may provide evidence of a predisposition to vasovagal syncope or they may implicate a variety of alternative causes for reported symptoms. However, the definitions of such abnormal responses have been largely anecdotal, as no comprehensive normal data have been provided for the prolonged durations currently used in diagnostic tilt testing.
HEART RATE BEHAVIOUR

The normal heart rate behaviour during tilt involves an early rise, with an immediate increase predominantly caused by withdrawal of vagal tone, and a more delayed increase over the first two minutes caused by enhanced sympathetic activity. ${ }^{38} 39$ The magnitude of the early increase is age related. ${ }^{39}$ The subsequent heart rate behaviour has not been described in detail for this tilt protocol, although some early reports and two more recent studies showed a smaller progressive rise throughout the duration of a variety of tilt protocols. ${ }^{9}{ }^{29}$ The data presented in this study reproduced the age related early increases in heart rate and confirmed that at all ages a further progressive rise continues throughout the tilt.

Excessive heart rate increases in the upright posture and during tilt may precede vasovagal syncope and predict successful treatment with $\beta$ blockers. $^{3537}$ Sinus tachycardia without hypotension may also be associated with dizziness, sweating, and palpitations and constitute the orthostatic tachycardia syndrome. ${ }^{36}{ }^{41}$ This may result from hypovolaemia with secondary sympathetic activation, and some researchers have preferred the term "idiopathic hypovolaemia." 36 Alternatively the response may occur as a result of a selective sympathetic neuropathy leading to excessive peripheral pooling of blood on assuming the upright position. ${ }^{42}$

Abi-Samra and colleagues defined an exaggerated chronotopic response during $60^{\circ}$ head up tilt as an increase of $30 \%$ and 20 beats $/ \mathrm{min}$ over baseline heart rate, and observed this "abnormality" in $35 \%$ of 154 patients. ${ }^{3}$ The VASIS classification also recognised a response involving an "excessive heart rate rise", suggesting that an increase of heart rate above 130 beats/min preceding vasovagal syncope may indicate a different underlying mechanism. ${ }^{5}$ Applying the criteria of Abi-Samra and colleagues to the normal population described in the present study, $47 \%$ of all normal subjects would be defined as abnormal. In contrast, the VASIS criteria may be unduly conservative as only $1 \%$ of normal individuals had a maximum heart rate of greater than 120 beats/min. For this protocol an increase in heart rate over the resting level of more than 45 beats/min for the $<35$ years age group, more than 40 beats/min for the 35-69 years group, and more than 30 beats $/ \mathrm{min}$ for the $70+$ years group occurs in $<2.5 \%$ of subjects, and these values represent appropriate criteria for an excessive rise.

Similarly it has been suggested that absent or inadequate chronotopic responses may identify patients with impaired orthostatic tolerance because of underlying intrinsic sinus node disease or autonomic dysfunction. ${ }^{35}$ With this protocol, a failure to increase the heart rate over the resting level by more than 10 beats/min for the $<35$ years age group and by more than 3 beats/min for the 35-69 and 70+ age groups occurs in $<2.5 \%$ of subjects, and these values represent appropriate criteria for an abnormally small rise. 
BLOOD PRESSURE BEHAVIOUR

In most studies of normal subjects during tilt, blood pressure has been monitored with manual or automatic sphygmomanometry. No change-or a limited reduction-in systolic blood pressure, and a progressive increase in diastolic pressure, have usually been reported..$^{13} 30$ In this study, blood pressure was monitored using a digital photoplethysmographic technique (Finapres). ${ }^{24} 44$ This method allows continuous and non-invasive measurements of blood pressure. It has been evaluated both in short term tilt methods and during the protocol used in this study. ${ }^{25}{ }^{45}$ In comparison with intra-arterial blood pressure measurements this technique is subject to considerable differences in absolute pressures. Despite this reservation, changes in pressure are closely reproduced. ${ }^{25} 46-48$ As the clinical requirements of blood pressure monitoring during tilt are primarily the identification and relative timing of such changes, without influencing outcome, the method seems well suited to diagnostic tilt tests.

The early increase in systolic blood pressure in each age group observed in this study is at variance with earlier observations. The difference probably reflects the distal site at which the pressure measurements were obtained and the established physiological changes which occur during tilt. With more distal measurements, systolic pressure amplification would be expected owing to waveform reflection in the peripheral circulation. ${ }^{49}$ This phenomenon is exaggerated by peripheral vasoconstriction and increasing heart rate, both of which occur during tilt. ${ }^{383950}$ Similar changes in systolic pressure have been noted in the first 60 seconds of head up tilt, a period during which intense finger vasoconstriction is known to occur. ${ }^{45}{ }^{51}$ The same phenomena are likely to explain the progressive increase in systolic pressure observed in some of the more elderly subjects during the latter stages of tilt.

Given the different method used for blood pressure measurement in this study it would be invalid to use the observed normal responses to evaluate the criteria for non-syncopal abnormalities of blood pressure behaviour described in patient populations. ${ }^{34}$ However, for this protocol, and employing the same method for blood pressure measurements, a fall in systolic pressure from resting values of more than $35 \mathrm{~mm} \mathrm{Hg}$ for subjects younger than 70 years, and of more than $50 \mathrm{~mm} \mathrm{Hg}$ for subjects aged $70+$ years, would represent $<2.5 \%$ of controls. Similarly a fall in diastolic pressure from resting values of more than $8 \mathrm{~mm} \mathrm{Hg}$ for subjects younger than 70 years, and of more than $12 \mathrm{~mm} \mathrm{Hg}$ for subjects aged 70+ years, would represent $<2.5 \%$ of controls.

\section{CONCLUSIONS}

In 1945, Allen stated, "There is at present no reliable means of identifying fainters other than the demonstration of fainting in the tilt board test." " To date this remains true, and tilt testing is used with increasing enthusiasm in the investigation of syncope. However, the results of this study show that false positive outcomes are likely to represent an important proportion of those observed in patients of all ages, and the recommended criteria for excessive heart rate responses during non-syncopal outcomes are largely invalid.

The more extreme forms of cardioinhibitory vasovagal syncope were rarely observed in the normal population, and such outcomes in patients are likely to be abnormal. Symptomatic postural hypotension, dysautonomic responses, and psychogenic syncope were not observed in normal subjects and can be considered abnormal. Caution is necessary in interpreting the more prevalent classical vasovagal outcomes. It remains to be established whether these conclusions can be extrapolated to other passive tilt test protocols.

MEVP was supported by the Garfield Weston Trust.

1 Kapoor WN, Smith MA, Miller NL. Upright tilt testing in evaluating syncope: a comprehensive literature review. $\operatorname{Am} \mathcal{F}$ Med 1994;97:78-88.

2 Kenny RA, Ingram A, Bayliss J, et al. Head up tilt: a useful test for investigating unexplained syncope. Lancet 1986;ii: 1352-4.

3 Abi-Samra F, Maloney JD, Fouad-Tarazi FM, et al. The usefulness of head up tilt testing and hemodynamic investigations in the workup of syncope of unknown origin. $P A C E$ 1988;11:1202-14.

4 Wahbha MMAE, Morley CA, Al-Shamma YMH, et al. Cardiovascular reflex responses in patients with unexplained syncope. Clin Sci 1989;77:547-53.

5 Sutton R, Petersen MEV, Brignole M, et al. Proposed classification for tilt induced vasovagal syncope. Eur $\mathcal{f}$ Cardiac Pacing Electrophysiol 1992;2:180-3.

6 Hackel A, Linzer M, Anderson N, et al. Cardiovascular and catecholamine responses to head up tilt in the diagnosis of recurrent unexplained syncope in elderly patients. $\mathcal{F} \mathrm{Am}$ Geriatr Soc 1991;39:663-9.

7 Petersen MEV, Williams TR, Sutton R. Psychogenic syncope diagnosed by prolonged head up tilt testing. $Q \mathcal{F}$ Med 1995;88:209-13.

8 Graybiel A, McFarland RA. The use of the tilt table test in aviation medicine. F Aviat Med 1941;12:194-211.

9 Allen SC, Taylor CL, Hall VE. A study of orthostatic insufficiency by the tiltboard method. Am $\mathcal{F}$ Physiol 1945;143: $11-20$.

10 Benditt DG, Remole S, Bailin S, et al. Tilt-table testing for evaluation of neurally mediated (cardioneurogenic) syncope: rationale and proposed protocols. PACE 1991;14: 1528-37.

11 Kapoor WN, Brant N. Evaluation of syncope by upright tilt testing with isoproterenol: a non-specific test. Ann Intern Med 1992;116:358-63.

12 Cheng TO. Need for standardisation of tilt-table test evaluation of syncope. Am $\mathcal{F}$ Cardiol 1992;69:1639.

13 Stevens PM. Cardiovascular dynamics during orthostasis and the influence of intravascular instrumentation. Am $\mathcal{F}$ Cardiol 1966;17:211-18.

14 Fitzpatrick AP, Theodorakis G, Vardas P, et al. Methodology of head up tilt testing in patients with unexplained syncope. f Am Coll Cardiol 1991;17:125-30.

15 Natale A, Akhtar M, Jazayeri M, et al. Provocation of hypotension during head up tilt testing in subjects with no history of syncope or presyncope. Circulation 1995;92:54-8.

16 Sander-Jensen K, Secher NH, Astrup A. Hypotension induced by passive head up tilt: endocrine and circulatory mechanisms. Am $\mathcal{F}$ Physiol 1986;251:R742-8.

17 Strasberg B, Rechavia E, Sagie A, et al. The head up tilt test in patients with syncope of unknown origin. Am Heart $\mathcal{f}$ 1989;118:923-7.

18 Raviele A, Gasparini G, Di Pede F, et al. Usefulness of head up tilt test in evaluating patients with syncope of unknown
origin and negative electrophysiologic study. Am 7 Cardiol origin and negative

19 Brignole M, Menozzi C, Gianfranchi L, et al. Neurally mediated syncope detected by carotid sinus massage and head up tilt test in sick sinus syndrome. Am f Cardiol 1991; 68:1032-6.

20 Grubb BP, Temesy-Armos PN, Hahn H, et al. Utility of upright tilt-table testing in the evaluation and management of syncope of unknown origin. Am f Med 1990;90:6-10.

21 Brignole M, Menozzi C, Gianfranchi L, et al. Carotid sinus massage, eyeball compression, and head up tilt in patients with syncope of uncertain origin and in healthy control subjects. Am Heart f 1991;122:1644-51.

22 Murray RH, Bowers JA, Goltra ER. Comparison of footboard and saddle supports for orthostatic tests on a tilt table. FAppl Physiol 1966;21:1409-11.

23 Kligfield P. Tilt table for the investigation of syncope: Is Kligfield P. Tilt table for the investigation of syncope: Is
there nothing simple about fainting? $\mathcal{f} \mathrm{Am}$ Coll Cardiol there nothing sim $1991 ; 17: 131-2$.

24 Penaz J. Photoelectric measurement of blood pressure, volume and flow in the finger. In: Digest of 10th 
International Conference on Medical and Biological Engineering, Dresden 1973 p104(7-2)

25 Petersen MEV, Williams TR, Sutton $\mathrm{R}$. A comparison of non-invasive continuous finger blood pressure measurement (Finapres) with intra-arterial pressure during prolonged head up tilt. Eur Heart f 1995;16:1647-54.

26 Shvartz E. Reliability of quantifiable tilt table data. Aerospace Med 1968;39:1094-8.

27 Shvartz E. Meyerstein N. Tilt tolerance of young men and young women. Aerospace Med 1970;41:253-5.

28 Davies R, Slater JDH, Forsling ML, et al. The response of arginine vasopressin and plasma renin to postural change in normal man, with observations on syncope. Clin Sci Mol Med 1976;51:267-74.

29 Kalbfleisch JH, Reinke JA, Porth CJ, et al. Effect of age on circulatory response to postural and valsalva tests. Proc Soc Exp Biol Med 1977;156:100-3.

30 Hainsworth R, Al-Shamma YMH. Cardiovascular responses to upright tilting in healthy subjects. Clin Sci 1988 ; 74:17-22.

31 Lipsitz LA, Mietus J, Moody GB, et al. Spectral characteristics of heart rate variability before and during postural tilt: relations to ageing and risk of syncope. Circulation 1990;81 $1803-10$

32 Kaufmann H, Oribe E, Oliver JA. Plasma endothelin during upright tilt: relevance for orthostatic hypotension. Lancet 1991;338:1542-5.

33 Shalev Y, Gal R, Tchou PJ, et al. Echocardiographic demonstration of decreased left ventricular dimensions and vigorous myocardial contraction during syncope induced by head up tilt. F Am Coll Cardiol 1991;18:746-51.

34 Lewis $T$. Vasovagal syncope and the carotid sinus mechanism: with comments on Gower's and Northnagel's syndrome. BMF 1932;i:873-6.

35 Petersen MEV, Williams T, Sutton R. A prospective evaluation of tilt test outcomes using continuous non-invasive beat-to-beat blood pressure and heart rate monitoring [abstract]. Eur f Cardiac Pacing Electrophysiol 1994;4:150. [abstract]. Eur

36 Jaeger FJ, Maloney JD, Castle LW, et al. Is absolute hypovolaemia a risk factor for vasovagal response to head up tilt?

37 Leor J, Rotstein Z, Vered Z, et al. Absence of tachycardia during tilt test predicts failure of $\beta$-blocker therapy in patients with neurocardiogenic syncope. Am Heart f 1994 127:1539-43.
38 Borst C, Wieling W, van Brederode JFM, et al. Mechanisms of initial heart rate response to postural change. Am $\mathcal{f}$ Physiol 1982;243:H676-81.

39 Dambrink JHA, Weiling W. Circulatory response to postural change in healthy male subjects in relation to age. Clin Sci 1987;72:335-41.

40 Hellebrandt FA, Franseen EB. Physiological study of the vertical stance in man. Physiol Rev 1943;23:220-5.

41 Hoeldtke RD, Davis K. The orthostatic tachycardia syndrome: evaluation of autonomic function and treatment with octreotide and ergot alkaloids. F Clin Endocrinol Metab 1991;73:132-9.

42 Oh JJ, Kim CH. Gastroparesis after a presumed viral illness: clinical and laboratory features and natural history. Mayo Clin Proc 1990;65:636-42.

43 Molhoek GP, Wesseling KH, Settels JJ. Evaluation of the Penaz servo-plethysmo-manometer for the continuous, non-invasive measurement of finger blood pressure. Basic Res Cardiol 1984;79:598-609.

44 Boehmer RD. Continuous real-time, non-invasive monitor of blood pressure: Penaz methodology applied to the finger. f Clin Monit 1987;3:282-7.

45 Imholz BPM, Settels JJ, van der Meiracker AH, et al. Noninvasive continuous finger blood pressure measurement during orthostatic stress compared to intra-arterial pressure. Cardiovasc Res 1990;24:214-21.

46 Kermode JL, Davis NJ, Thompson WR. Comparison of the Finapres blood pressure monitor with intra-arterial manometry during the induction of anaesthesia. Anaesth Intensive Care 1989;17:470-86.

47 Epstein RH, Bartkowski RR, Huffingdale S. Continuous noninvasive finger blood pressure during controlled hypotension. Anaesthesiology 1991;75:796-803.

48 Farquhar IK. Continuous direct and indirect blood pressure measurement (Finapres) in the critically ill. Anaesthesia 1991:46:1050-5.

49 O'Rourke MF, Blazek JV, Morreels CL, et al. Pressure wave transmission along the human aorta; changes with age and in arterial degenerative disease. Circ Res 1955;3:623-31.

50 Rowell IB Brengelmann G Disparities between aortic and peripheral pulse pressure Disparities between aortic and peripheral pulse pressure
induced by upright exercise and vasomotor changes in induced by upright exercise an

51 Beaconsfield P, Ginsburg J. The effect of body posture on the hand blood flow. F Physiol (Lond) 1955;130:467-73.

\section{IMAGES IN CARDIOLOGY}

\section{Isolated left atrial tamponade following circumflex artery angioplasty}

An 80 year old man presented with unstable angina four weeks after undergoing two vessel coronary artery bypass surgery. Percutaneous transluminal coronary angioplasty of an ungraftable circumflex artery was complicated by a coronary artery perforation. The patient was initially stable but after several hours developed haemodynamic instability. Transthoracic and later intraoperative transoesophageal echocardiography revealed a large isolated haematoma in the oblique sinus compressing the posterior left atrium (A, short axis view through the aortic valve; $\mathrm{AO}$, aortic valve; LA, left atrium; RA, right atrium; $\mathrm{TH}$, thrombus). An emergency left posterior left thoracotomy was performed and the thrombus evacuated with relief of the left atrial compression (B). No further cardiac intervention was required and the patient was successfully discharged.

ALEXANDER KADNER

RAYMOND H CHEN CHARLES D COLLARD DAVID H ADAMS

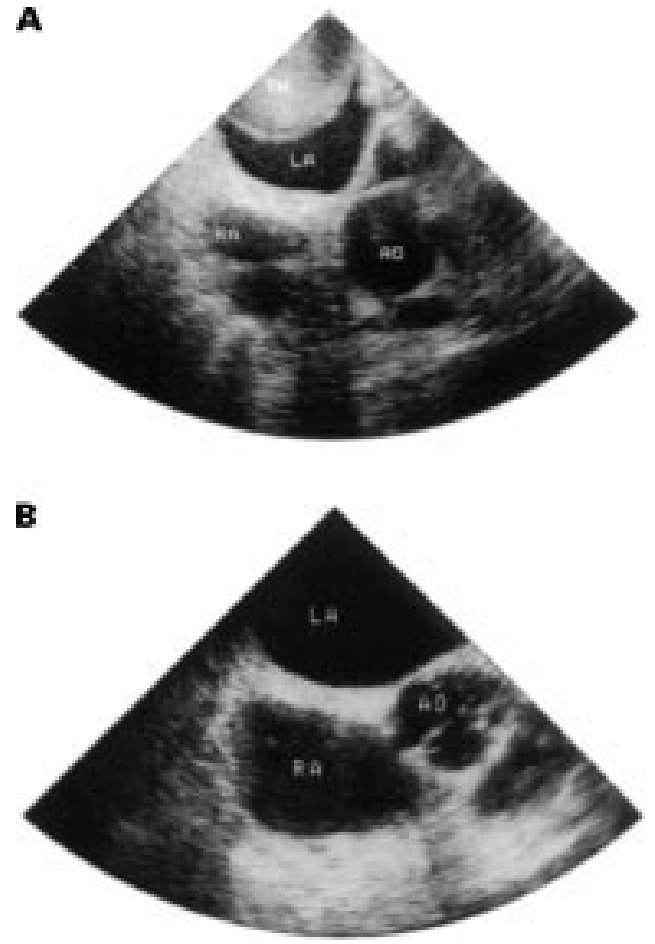

